\section{Advantages of general practice}

SIR - Barbara J. Culliton argues that a 50 per cent quota of medical students earmarked for general practice is unlikely to help the US health-care crisis (Nature 370, $501 ; 1994)$. Her damaging allegations about health systems based on primary care must not go uncontested.

I challenge her to cite valid epidemiological references to support the claim that because of a lower proportion of specialists, "people in Britain are more likely to die of cancer than their counterparts in the United States and Europe". Any such 'evidence' is more likely the result of inappropriate 'screening' leading to earlier detection but no benefit (and in some cases discovery of disease that would never be clinically manifest). Enhanced 'survival' entails living longer with the diagnosis rather than more effective treatment or real extension of life. Her figures presumably also omit the millions in the United States excluded from the 'skilled specialist' Utopia because of inadequate insurance cover (U. E. Reinhardt, New Engl. J. Med. 330, 1452-1453; 1994).

More importantly, she neglects the role of the primary-care physician in selecting the specialist a patient sees. A subject with abdominal pain might refer herself to an accident and emergency department, urologist, gynaecologist, venereologist or gastroenterologist, and receive different high-technology investigations from each. Even if the specialist rapidly recognizes a condition outside his or her field, appropriate referral is delayed. Similarly, if an uncomplicated urinary tract infection were the cause, the primary-care physician could make the diagnosis sooner, and be capable of prescribing appropriate therapy at a fraction of the expense.

More insidiously, the 'worried well' or those with benign self-limiting conditions are subjected to stereotypical 'full diagnostic work-ups'. The nature of many of these investigations, validated only in the severely unwell, yields a steady supply of borderline abnormals, incidental findings, false positives and artefacts. These in turn ensure further rounds of interventions.

The patient is protected from this if seen first by a primary-care physician with a broad knowledge of the patient's medical history and economical, emotional, psychological or spiritual backgrounds of which Culliton speaks so disparagingly. She shares an attitude born of uncritical attendance at too many medical school case presentations; there symptoms are inevitably due to esoteric yet highly treatable organic conditions missed by blundering generalists. I suggest it is within those ivory towers that she should look for the "physicians as God" she cites, convinced of the omnipotence of hightechnology medicine. Primary care physi- cians are best placed to ensure simple preventive measures and behavioural changes. These can save and enhance far more lives than continued overprovision of specialists, many of whom make little or no contribution to basic or clinical science.

Research suffers on both sides of the Atlantic from administrations bent on short-term minimization of cost rather than long-term maximization of value. But Culliton has done the biomedical community a disservice with her transparently biased and elitist rhetoric.

Malcolm H. Duncan

Department of Chemical Pathology,

Charing Cross and Westminster

Medical School,

Fulham Palace Road,

London W6 8RF, UK

SIR - Culliton, in arguing that primary care is not the answer to the health-care problem in the United States, fails to mention an important fact. Total spending on health care in the United States is about 14 per cent of gross domestic product (GDP) and about $\$ 2,000$ per capita, yet at least 35 million people have no cover. The author contrasts the system in the United Kingdom unfavourably with that in the United States. In the United Kingdom we spend 7 per cent of our GDP (about $£ 600$ per capita) and there is complete coverage of the population.

Health care in the United Kingdom seems to be more 'efficient' than that in the United States even though it is hampered by inadequate funding. A major difference between the two systems is that in the United States the patient must perform a preliminary diagnosis and seek out the appropriate specialist. Consideration of Bayes' theorem indicates that the predictive value of a test depends upon the prevalence of the condition in the population (T. Bayes, 'Essay towards solving a problem in the doctrine of chances', Phil. Trans. R. Soc., 1763.) A specialist who is used to seeing a population in which the proportion of people with coronary artery disease is high will thus tend to overdiagnose the condition when exercising the same clinical skills upon a population where the prevalence of disease is low. This is because specialists' clinical judgement and the tests upon which they rely are 'tuned' to the population they normally see.

A man who is depressed and worried about a muscular pain in his chest may choose to see a cardiologist who diagnoses ischaemic heart disease. The patient may then have further tests that lead to major heart surgery. The operation may be expertly performed and technically very successful, but, if the main problem of depression persists, it is difficult to argue that the man has received "high quality health care".

Human beings are more than the sum of the functions of their limbs and organs. Many consultations with physicians have a psychological or social component that presents as physical illness and can lead to unnecessary or harmful investigation.

Comparisons between the health-care systems of different countries are fraught with problems. Health has less to do with doctors and health care than many imagine, and more to do with the social and economic circumstances of the individual. But by most parameters the UK healthcare system ranks highly and the relationship in the National Health Service between primary and secondary care probably deserves some credit for this.

Modern biomedical research is essential and we should be quick to evaluate it and make its benefits available to the general population. The problem with the UK system of health care is not with its structure but with the lack of funding. It is most efficient to use specialist resources on a population where the prevalence of 'real' illness is high. This means that the doctor the patient first sees must be expert in identifying those who will benefit from specialist help. A well-educated, wellsupported primary-care physician is the key to the efficient delivery of high quality, high technology health care.

\section{Robert Walton}

Godfrey Fowler

University of Oxford,

Radcliffe Infirmary,

oxford OX2 $6 H E$, UK

\section{Apoptosis: two p or not two p?}

SIR - Attention is being paid to the correct pronunciation of the coined word apoptosis, for programmed cell death. Clearly the ' $p$ ' in ptosis is silent, and on this basis students are commonly exhorted to pronounce apoptosis as apo'tosis.

The silent ' $p$ ', however, appears neither correct nor attractive in words in which the Greek-derived 'pt' occurs in the middle of a composite word. Consider, for example, two words containing the Greek word for wing (pterodactyl, helicopter), in which the 'pt' is pronounced quite differently. In Italian, by contrast, ' $\mathrm{p}$ ' is neither written (nor sounded) in the equivalent words.

This suggests that students who sound both 'p's in apoptosis may be correct, and that adenoidal attempts by their elders to suppress the second 'p' are not well grounded in etymology.

\section{John Funder}

BakerMedical Research Institute,

POBox 348, Prahran,

Victoria 3181, Australia 